\title{
Necrotizing Enterocolitis Totalis
}

National Cancer Institute

\section{Source}

National Cancer Institute. Necrotizing Enterocolitis Totalis. NCI Thesaurus. Code C114909.

A severe and often fatal form of necrotizing enterocolitis, in which diffuse ischemia, necrosis, and pneumatosis intestinalis are evident in the small and large intestine. Short bowel syndrome is common among survivors. 